\title{
Relación entre el Dinamismo Percibido, la Postura Tecnológica y los Resultados de Innovación
}

\section{Relationship among Perceived Dynamism, Technological Posture and Innovation Performance}

\author{
María Isabel González-Ramos', Mario Javier Donate-Manzanares², Fátima Guadamillas-Gómez³, Joaquín \\ Alegre-Vidal ${ }^{4}$
}

\begin{abstract}
Una de las principales elecciones estratégicas de la empresa es su postura tecnológica, entendida como su propensión innovadora y el momento de introducción de cambios tecnológicos en el mercado. Este trabajo, centrado en el sector de las energías renovables en España, plantea que en entornos en los que el dinamismo percibido por los directivos es alto y la apreciación del cambio mayor, las empresas tienen una mayor tendencia hacia el liderazgo tecnológico (entrada pionera) en detrimento de posturas más conservadoras o de seguimiento tecnológico (entrada tardía). Los resultados del análisis, que se ha llevado a cabo mediante un modelo de ecuaciones estructurales basado en la técnica de mínimos cuadrados parciales (PLS), muestran que, efectivamente, el dinamismo percibido está positivamente relacionado con el liderazgo tecnológico, y que esta relación provoca un fuerte efecto sobre los resultados de innovación en términos de generación de nuevas tecnologías tanto en productos como en procesos.
\end{abstract}

Keywords: dinamismo percibido; postura tecnológica; resultados de innovación; sector de energías renovables; españa.

A main strategic election for a firm is its technological posture, which can be understood as its propensity to innovate and the moment for the introduction of technological changes into the market. This paper, which focuses on the renewable energy industry in Spain, establishes that in environments in which dynamism is understood by managers as high, and the appreciation of change is greater, firms develop a greater tendency towards technological leadership postures (pioneer or first-mover strategy) rather than conservative or follower postures (last-mover strategy). The results of the analysis, carried out through a structural equation model based on the partial least squares (PLS) technique, show that, as anticipated, perceived dynamism is positively related to technological leadership (first-mover strategy), and that this relationship has a strong effect on innovation performance, in terms of generation of new technologies both in products and processes.

Keywords: perceived dynamism; technological posture; innovation performance; renewable energy sector; spain.

\footnotetext{
1,3 Departamento de Administración de Empresas, Facultad de Ciencias Jurídicas y Sociales, Universidad de Castilla-La Mancha. Cobertizo San Pedro Mártir s/n, 4507। Toledo, España.Tel: +34 925268800 (ext. 516I); Fax: +34 902204 I 30 e-mail: 'Mariaisabel.gonzalez@uclm.es (María Isabel González, correspondencia autor principal).

2Departamento de Administración de Empresas, Facultad de Derecho y Ciencias Sociales, Universidad de Castilla-La Mancha. ${ }^{4}$ Departamento de Dirección de Empresas 'Juan José Renau Piqueras', Universidad de Valencia.
}

ISSN: 07 I 8-2724. (http://www.jotmi.org) 


\section{Introducción}

Las características de globalización y cambio continuo que definen el entorno empresarial actual provocan que la capacidad de adaptación continua de la empresa sea una condición necesaria para su supervivencia y desarrollo (Jansen, Vera y Crossan, 2009). En este sentido, muchas empresas desarrollan estrategias de innovación proactivas, con el objetivo de hacer frente a la rapidez y profundidad de los cambios que acontecen a su alrededor (Eisenhardt y Martin, 2000; Bierly y Daly, 2007). El dinamismo del entorno, por tanto, puede considerarse una variable relevante a la hora de tomar decisiones estratégicas sobre innovación así como el momento del tiempo en que la tecnología tendrá un impacto más significativo para la empresa por su entrada en el mercado (Suárez, 2004; Min, Kalwani y Robinson, 2006).

Aunque la elección de la agresividad tecnológica y el momento de entrada en el mercado en ámbitos innovadores (por ejemplo, sectores de alta tecnología) son decisiones muy relevantes desde el punto de vista estratégico, la investigación existente no deja claro qué postura puede resultar más ventajosa para la empresa (primer entrante, seguidor, entrante tardío) en términos del impacto de los resultados de la innovación obtenidos (Schilling, 2012). En este trabajo, y siguiendo una perspectiva de capacidades dinámicas (Teece, Pisano y Shuen, 1997), planteamos que la forma de percibir el dinamismo del entorno por parte de la alta dirección tiene una influencia directa en la elección de la postura tecnológica, lo que a su vez impactará de forma determinante en los resultados obtenidos en innovación.

En este sentido, los trabajos previos que han analizado la relación entre alguno de los componentes de la estrategia de innovación (la postura tecnológica es uno de ellos) y los resultados han considerado con frecuencia al dinamismo del entorno como una variable moderadora o contingente (contextual) (Spital y Bickford, 1992; Zahra, 1996; Zahra y Bogner, 1999; Bierly y Daly, 2007; Jansen, Vera y Crossan, 2009). Es decir, han considerado que el grado de dinamismo que presenta el entorno no condiciona la elección de una determinada estrategia de innovación, sino que afecta a la relación entre ésta y los resultados empresariales. Por el contrario, pocos autores han contemplado una relación directa entre el dinamismo del entorno y la elección de la estrategia en términos tecnológicos. Una excepción es el trabajo de Su y Chiu (2004), quienes señalan que cuando el entorno competitivo se presenta dinámico, las empresas que destinan más recursos a $1+D+i$ tienen más probabilidades de desarrollar una adecuada estrategia competitiva a través de la tecnología. Por su parte, Zahra (1996) plantea que en entornos altamente dinámicos, moderadamente hostiles y moderadamente heterogéneos, la tendencia hacia el desarrollo de una postura de liderazgo tecnológico por parte de la empresa es mayor que en entornos que cuentan con características diferentes, sobre todo en lo que se refiere a dinamismo ambiental.

De forma similar, en este trabajo se establece que el grado de dinamismo percibido del entorno es una variable muy relevante a la hora de tomar decisiones sobre postura tecnológica en actividades relacionadas con las energías renovables, ya que por una parte, la tecnología y la innovación son variables clave en este sector de actividad. Por tanto, las decisiones sobre la orientación tecnológica son esenciales para optimizar los esfuerzos llevados a cabo en I+D+i. Por otra parte, el ámbito de las energías renovables incluye un conjunto de actividades heterogéneas, tanto desde el punto de vista tecnológico como de mercado, con múltiples industrias afines, auxiliares y complementarias a la actividad principal (producción de energía renovable). Esta heterogeneidad de actividades implica que las decisiones sobre el desarrollo y la introducción en el mercado de nuevos productos o procesos no son sencillas y dependen fuertemente de las percepciones de los directivos sobre la evolución de variables como la intensidad de la competencia o de aspectos tecnológicos, políticos y legales.

A diferencia de otras investigaciones previas que han considerado múltiples sectores de actividad (Covin, Slevin y Heeley, 1999;Wijbenga y Van Witteloostuijn, 2007; Wilden et al., 20I3), en este trabajo se emplea una muestra de empresas pertenecientes exclusivamente al sector de las energías renovables en España por múltiples motivos. En primer lugar por los beneficios de tratar con empresas que probablemente son homogéneas desde un punto de vista tecnológico, aún con el riesgo de no alcanzar conclusiones generalizables al resto de sectores (Fernández-Mesa,Alegre-Vidal y Chiva-Gómez, 2012). En segundo lugar porque se trata de un sector dinámico e innovador. Según datos del informe APPA (20I I), la inversión de este sector en I+D+i en el año 2010 supuso un $4.5 \%$ de su contribución al PIB, mientras que la media nacional en ese mismo año se situó en el I.38\%.Y en tercer lugar por la gran importancia que tiene este sector para la economía española, ya que se trata de una industria en crecimiento cuya contribución al PIB en el año 2012 estuvo en torno al $\mathrm{I} \%$.

Así pues, el objetivo específico de este trabajo y su principal aportación es determinar si el grado de dinamismo que los directivos perciben del entorno condiciona la postura tecnológica adoptada por la empresa y los resultados de innovación en el sector de las energías renovables en España (véase figura I en la pág. I5). La estructura de desarrollo de este artículo es la siguiente. En primer lugar, se presenta de forma teórica la relación entre el dinamismo del entorno, la postura tecnológica y los resultados de innovación de la empresa. A continuación, se analiza empíricamente, a través 
de un modelo de ecuaciones estructurales al que se aplica la técnica de mínimos cuadrados parciales (PLS), la relación entre estas variables en una muestra de 76 empresas españolas pertenecientes al sector de las energías renovables. Para finalizar, se extraen algunas implicaciones prácticas y conclusiones sobre cómo la postura tecnológica puede convertirse en un factor clave para lograr mejores resultados innovadores si es coherente con la forma en que los directivos perciben la dinamicidad de su entorno.

Dinamismo del entorno y postura tecnológica de la empresa El tipo de entorno en el que la empresa desarrolla su actividad afecta a las elecciones de los directivos referentes a la estrategia tecnológica (Spital y Bickford, 1992; Bierly y Daly, 2007;Wijbenga y Van Witteloostuijn, 2007; Jansen, Vera y Crossan, 2009). Esta estrategia debe establecer el camino de desarrollo de los recursos tecnológicos que permitan a la empresa aprovechar las oportunidades del mercado y contrarrestar la incertidumbre, la cual depende fuertemente del nivel de dinamismo que presenta el entorno (Zahra, 1996). Para algunos autores, el dinamismo del entorno depende de la intensidad, regularidad e imprevisibilidad con la que se presentan los cambios en un sector de actividad, el comportamiento imprevisible de los clientes, los cambios en sus necesidades y preferencias, la entrada de nuevos competidores o los cambios en las condiciones tecnológicas del sector, entre otros factores (Bierly y Daly, 2007; Jansen, Vera y Crossan, 2009). Por ejemplo, para Spital y Bickford (1992), el dinamismo del entorno depende fundamentalmente de la rapidez de los cambios que se producen en las tecnologías que incorporan los productos o de los avances tecnológicos en los procesos, por lo que básicamente consideran el dinamismo en función de los cambios en las condiciones tecnológicas del sector de actividad.

Otros autores consideran que un entorno dinámico se caracteriza por grandes y rápidos cambios en las preferencias de los consumidores y en los productos ofertados por los productores, lo que implica que las preferencias de los consumidores y las acciones de los competidores son mucho más difíciles de predecir en entornos dinámicos que en entornos estables (Wijbenga y Van Witteloostuijn, 2007). El grado de previsibilidad es, pues, una característica importante a la hora de distinguir entre entornos estables y dinámicos, considerando que los entornos dinámicos son menos previsibles porque los cambios que surgen en las preferencias de consumidores y productores ocurren de forma más brusca y menos gradual que en los entornos estables (Eisenhardt, Furr y Bingham, 20I0).

El dinamismo del entorno es una variable que tiene implicaciones sobre numerosas decisiones estratégicas de la empresa (Jansen, Vera y Crossan, 2009; Eisenhardt, Furr y Bingham, 20I0). Por ejemplo, debido a la obsolescencia tec- nológica que provoca, algunas compañías deciden aumentar sus esfuerzos en I+D+i para desarrollar nuevos productos, otras llevan a cabo alianzas estratégicas con la intención de tener acceso a mayor número de innovaciones tecnológicas, y otras adquieren empresas de base tecnológica para aumentar sus esfuerzos en el desarrollo interno de $1+D+i$ (Zahra, 1996: 192).

Los entornos dinámicos pueden fomentar que las empresas adopten una postura de liderazgo tecnológico con el objetivo de identificar mercados emergentes, lanzar nuevos productos en mercados existentes, y adelantarse a la entrada de nuevos competidores (Guerras y Navas, 2007). Además, mediante un comportamiento proactivo, el pionero puede controlar la dirección de la evolución tecnológica del sector (Suárez, 2004). Mientras que la evolución tecnológica suele ser impredecible, el pionero puede guiar los cambios en el estándar tecnológico creando nuevas tendencias o modificando las ya existentes (Schilling, 20 I2; Hawk, Pacheco-DeAlmeida y Yeung, 2013).

Sin embargo, también existen investigaciones que aportan evidencias en sentido contrario. Por ejemplo, Suárez y Lanzolla (2007) argumentan que el dinamismo del entorno es perjudicial para el mantenimiento de las ventajas competitivas del pionero, siendo preferible optar por un comportamiento imitador o seguidor en entornos turbulentos. Otros argumentan que un dinamismo del entorno extremo puede acelerar la obsolescencia del producto y reducir la rentabilidad de las nuevas tecnologías, provocando que los beneficios de la mayor parte de las empresas sean efímeros (Bierly y Daly, 2007). Por otra parte, también puede no compensar la participación simultánea de las empresas en el desarrollo de numerosas tecnologías, obligándolas a centrarse solamente en aquellas innovaciones tecnológicas en las que puedan destacar por encima de sus competidores, o lo que es lo mismo, se pueden presentar oportunidades para los pequeños competidores, que desarrollarán nuevas tecnologías y se beneficiarán de su mayor flexibilidad para introducirlas rápidamente en el mercado (Bierly y Daly, 2007). Sin embargo, hay que tener en cuenta que, aunque un elevado grado de dinamismo puede cerrar ventanas de oportunidad existentes, también crea nuevos nichos de mercado y promueve la expansión de la cartera de productos y procesos tecnológicos de las empresas (Wijbenga y Van Witteloostuijn, 2007; Schilke, 2013).

Por otro lado hay que tener en cuenta que, incluso dentro de una misma industria o sector de actividad, las percepciones del entorno varían de una compañía a otra, ya que su orientación al riesgo, las destrezas directivas, recursos y capacidades, así como sus resultados anteriores son diferentes, por lo que van a condicionar dicha percepción (Zahra, 1996; Schilke, 2013). Por ello, este trabajo se centra especí- 
ficamente en el dinamismo del entorno percibido por los directivos de la empresa. Entendemos en este sentido que los directivos tomarán decisiones referentes a la estrategia tecnológica basándose, en parte, en la forma en que perciben la evolución del entorno y su efecto en la empresa en términos de dinamicidad (Jansen, Vera y Crossan, 2009; Eisenhardt, Furr y Bingham, 20I0).

La investigación existente en estrategia tecnológica destaca las características del entorno como un factor importante a considerar a la hora de establecer una determinada postura innovadora (e.g., Zahra, 1996; Covin, Slevin y Heeley, 1999; Bierly y Daly, 2007; Súarez y Lanzolla, 2007; Wijbenga y Van Witteloostuijn, 2007; Jansen, Vera y Crossan, 2009). Por lo tanto, los directivos deberían prestar atención a dichas características, $y$ en especial a su dinamicidad, a la hora de realizar la selección de la postura tecnológica (Spital y Bickford, 1992; Su y Chiu, 2004; Bierly y Daly, 2007). En general, a la hora de llevar a cabo dicha selección, los directivos deben considerar los objetivos de su empresa, la capacidad de la misma para liderar diversas innovaciones en producto y en proceso, su orientación al riesgo, sus recursos disponibles, y la posibilidad de adaptarse al entorno dependiendo de su dinamicidad (Schilke, 20I3).

Así pues, es probable que a medida que varíe la percepción del entorno por parte de los directivos, también lo haga la predisposición de la empresa hacia la búsqueda de una determinada postura tecnológica. Además, hay que señalar que en sectores intensivos en tecnología, como es el caso del sector de las energías renovables, quedarse rezagado y no tener en cuenta los cambios que se producen en el entorno puede significar perder posiciones en el mercado que pueden llegar a ser prácticamente irrecuperables (Lieberman y Montgomery, 1988; Jansen et al., 2009; Schilke, 2013). Por otra parte, $y$ desde un punto de vista evolutivo, la tecnología ha cambiado radicalmente el marco de la competencia entre empresas. En este sentido, el propio avance de las tecnologías es un factor externo que impulsa, $y$ en cierta medida obliga al establecimiento de estrategias proactivas con el objeto de permanecer en la brecha competitiva (Burgelman, Maidique yWheelwright, 200 I: 39-40; Gibbons y O'Connor, 2003). Por todo ello se formula la primera hipótesis de este trabajo estableciendo que:

Hipótesis I.A mayor dinamismo percibido del entorno, mayor la tendencia de la empresa a desarrollar una postura de liderazgo tecnológico.

\section{Liderazgo tecnológico y resultados de innovación}

La estrategia proactiva o de liderazgo tecnológico se apoya en una política dinámica en $I+D+i$, basada en la posibilidad de identificar nuevas necesidades del mercado y descubrir la manera de satisfacerlas (Schilling, 20I2; Schilke, 20I3). Una empresa pionera se define como aquella que encabeza su sector de actividad en cuanto a la creación de tecnologías que requieren innovaciones en producto $\circ$ en proceso (Zahra, 1996; Zahra y Bogner, 1999). El desarrollo de productos se ha convertido en una capacidad dinámica de las empresas, debido a su capacidad para alterar la configuración de recursos de las organizaciones (Eisenhardt y Martin, 2000). A menudo se relaciona la capacidad de una empresa para desarrollar nuevos productos con la consecución de ventajas competitivas (Teece, Pisano y Shuen, 1997; Schilke, 2013). Tomando como referencia el enfoque basado en los recursos (EBR) y el enfoque basado en las capacidades dinámicas (EBCD), es importante señalar que la capacidad para desarrollar innovaciones (en producto o en proceso) normalmente implica un compromiso de recursos a largo plazo que no todas las empresas pueden asumir. Por ello, aquellas que deciden comprometer suficientes recursos necesitan aprovechar sus capacidades dinámicas para intentar que las innovaciones generadas se traduzcan en ingresos que superen los costes de oportunidad en los que incurren (Schilke, 2013). Así pues, adoptar una postura de liderazgo tecnológico supone poseer un conjunto de capacidades tecnológicas internas que permitan conseguir ventajas competitivas (Spital y Bickford, 1992; Wilbon, 2003).

Por un lado, la innovación en producto es fundamental para la renovación de las empresas y para adelantarse a la competencia (Dougherty, 1992). Éstas dependen en gran medida de la introducción y comercialización de nuevos productos en entornos dinámicos, donde se produce una rápida obsolescencia de los productos y las necesidades de los consumidores cambian frecuentemente, lo que provoca que las empresas se enfrenten a una intensa competencia (Wilbon, 2003; Yang y Liu, 2006).

Las empresas tecnológicas buscan el crecimiento principalmente a través del desarrollo de nuevos productos. Sin embargo, estas empresas a veces tienen problemas para crecer, ya que la innovación continua puede ocasionar un efecto no deseado, exponiendo a los consumidores a la novedad de manera generalizada y haciendo que éstos se acostumbren a un flujo continuo de nuevos productos y servicios que quedan obsoletos rápidamente (Wilbon, 2003; Yang y Liu, 2006).

Por otro lado, las innovaciones en proceso generalmente favorecen la estrategia de diferenciación, ya que muchas de las mejoras que desarrollan los pioneros están orientadas hacia una mayor flexibilidad del proceso productivo con el objetivo de lograr una mayor personalización del producto (Porter, 1985). Sin embargo, en otros casos, las empresas persiguen el liderazgo tecnológico en proceso para desarrollar una estrategia de liderazgo en costes, jugando un papel 
fundamental en este caso la automatización del proceso y la consiguiente disminución del tiempo empleado para transformar inputs en outputs, lo cual reducirá el coste unitario del producto (Min, Kalwani y Robinson, 2006; Bierly y Daly, 2007; Jansen, Vera y Crossan, 2009). Así, mediante una estrategia de liderazgo tecnológico en proceso las empresas pueden mejorar los procesos de fabricación para entregar productos de calidad a un precio inferior, obteniendo con ello ventajas sobre la competencia (Parker, 2000).

En este artículo, se entenderá la postura de liderazgo tecnológico como una actitud pionera en la generación de tecnologías de producto o proceso, con el fin de adelantarse a la competencia en la entrada al mercado. Esta estrategia está basada, en este sentido, en la anticipación constante con el fin de aprovechar ventajas de primer entrante, frente a empresas que pueden tener preferencia por entrar de forma más tardía al mercado o carecer de las capacidades suficientes como para hacerlo de forma temprana (Min, Kalwani y Robinson, 2006).

Por lo general, no todas las empresas pueden poner en práctica una postura pionera, ya que se requiere habilidad para la innovación tecnológica así como capacidad para identificar nuevas oportunidades de mercado y traducirlas rápidamente en productos o procesos (Spital y Bickford, 1992; Haleblian et al., 2012). En este sentido, Pavitt (1990: 24) argumentó que la postura tecnológica adoptada por una empresa depende en gran medida del tamaño de la misma y de sus competencias tecnológicas acumuladas, lo que determinará conjuntamente su potencial tecnológico y las oportunidades de mercado que pueda explotar. De forma similar, Haleblian et al. (2012: 1048) consideran que los líderes tecnológicos suelen tener un mayor conocimiento del mercado que los seguidores, una mayor motivación para actuar y una capacidad de actuación también superior. Sin embargo, para algunos como Bierly y Daly (2007), cualquier empresa que adopte una postura seguidora, posea abundantes recursos y una gran capacidad de I+D+i puede imitar rápidamente los éxitos de los pioneros o incluso mejorarlos, y como consecuencia obtener también buenos resultados de innovación sin necesidad de ser la primera en entrar al mercado. Otros como Schilling (2012) afirman que, ante los cambios en las necesidades tecnológicas y en las preferencias de los consumidores, los seguidores tempranos tienen mayor capacidad que los pioneros para ofertar una gama de productos superior. Este hecho se justifica por la inercia ligada al comportamiento rutinario, característico de las empresas pioneras, que si bien las permite realizar sus actividades eficientemente, también limita su habilidad para cambiar.
Así pues, queda patente que existen diversas opiniones cuando se trata de establecer una relación entre postura tecnológica y resultados de innovación (en producto o en proceso). Sin embargo, basándonos en el EBR y el EBCD (Teece, Pisano y Shuen, 1997; Schilke, 2013), en este trabajo planteamos que los líderes tecnológicos poseen un conjunto de habilidades, competencias tecnológicas acumuladas y capacidades dinámicas que los seguidores no poseen (Spital y Bickford, 1992; Haleblian et al., 2012), lo que les otorga un mayor potencial tecnológico para explotar oportunidades de mercado y desarrollar innovaciones. Por ello, a continuación formulamos las siguientes hipótesis:

Hipótesis 2. A mayor tendencia hacia una postura de liderazgo tecnológico, mayores los resultados de innovación en producto de la empresa.

Hipótesis 3. A mayor tendencia hacia una postura de liderazgo tecnológico, mayores los resultados de innovación en proceso de la empresa.

\section{Metodología \\ Población y muestra}

Este estudio se ha llevado a cabo en empresas pertenecientes al sector de las energías renovables. En concreto, la población se divide en seis categorías: (I) compañías nacionales dedicadas a la producción de energía; (2) a la fabricación de componentes tecnológicos; (3) al desarrollo de actividades de ingeniería, soluciones integrales e innovación; (4) a la comercialización y exportación de dichos componentes; (5) consultoras energéticas y (6) empresas instaladoras y de mantenimiento. Sin embargo, estas seis actividades se pueden agrupar en sólo dos bloques, producción (las tres primeras) y servicios (las tres últimas) (ver tabla I).

El número total de empresas que pasaron a formar parte de nuestra población fue de 726. El estudio se realizó entre septiembre y diciembre de 2012 a través de cuestionario on-line a directivos de empresa o unidad de negocio y se recogieron datos referentes a los años 2010,2011 y 20I2. Tras contacto y seguimiento telefónico, se recogieron 76 cuestionarios válidos, que representan el $10.47 \%$ de tasa de respuesta. Este es un porcentaje aceptable ya que la literatura referente a management, como consecuencia del escaso incentivo que supone para las empresas contestar a este tipo de encuestas, considera válida una tasa de respuesta comprendida entre el 10 y el 20 por ciento en trabajos empíricos con recogida de datos a través de cuestionarios (véase por ejemplo Zahra y Bogner, 1999). El tamaño de las empresas que componen la muestra (medido por el número de empleados) así como la ficha técnica de la investigación se muestran en las siguientes tablas: 
Por último, al haber sido los datos recogidos en el mismo cuestionario y contar con un diseño transversal de investigación, podría darse el llamado problema de la varianza común, esto es, varianza que se atribuye al método de medida y que puede provocar un error de medida sistemático y distorsiones en la estimación de las verdaderas relaciones entre los constructos teóricos (Podsakoff y Organ, 1986). Para tratar de averiguar si existe este problema se ha aplicado el test de Harman (a través de un análisis factorial exploratorio). Para esta prueba, existiría un problema de varianza común si (a) surge sólo un único factor en el análisis, o (b) uno de los factores agrupa la mayoría de la varianza extraída (Bou-Llusar et al., 2009).

Por tanto, se ha aplicado un análisis factorial a través del método de componentes principales con rotación varimax al conjunto de ítems de la investigación, lo que ha revelado la existencia de 6 factores con autovalores mayores que 1.0 que agrupan el $77.12 \%$ de la varianza total.Además, el mayor factor agrupa el $24.52 \%$ de la varianza. Como no ha surgido un solo factor ni uno de los factores agrupa la mayoría de la varianza, se puede señalar que el problema de la varianza común no parece ser un problema grave en este trabajo y es improbable que pueda llevar a confusión en la interpretación de los resultados de la investigación (Podsakoff y Organ, 1986; Bou-Llusar et al., 2009).

\section{Medida de las variables}

A partir de la revisión de la literatura previa, se diseñaron una serie de indicadores para medir el dinamismo percibido del entorno, la postura tecnológica y los resultados de innovación, tanto en producto como en proceso. Cada uno de los ítems se estableció en base a una escala Likert (I "importancia concedida muy baja" y 5 "importancia concedida muy alta").

\section{Medida del dinamismo percibido del entorno}

En primer lugar, para medir la variable dinamismo percibido del entorno se analizó la forma en la que perciben los directivos de las empresas los cambios acontecidos en el entorno (Bierly y Daly, 2007). Para ello, se utilizó la misma escala de medida que Jansen, Vera y Crossan (2009) (ver anexo).

\section{Medida de la postura tecnológica}

Respecto a la medición de la postura tecnológica (siendo el criterio utilizado la actitud de la empresa con respecto a la creación y comercialización de nuevas tecnologías), se recogieron aspectos tales como ser la primera en introducir productos nuevos o mejorados en el mercado o incluso ganarse una reputación en el sector en cuanto a liderar cambios o mejoras en los productos (ver anexo). Esta escala de medida ha sido elaborada en base a los trabajos de Zahra y Das (1993), Zahra (1996) y Zahra y Bogner (1999).

\begin{tabular}{|c|c|c|c|c|c|}
\hline Caracterización de la muestra & $\begin{array}{c}\text { De I a 9 } \\
\text { empleados }\end{array}$ & $\begin{array}{c}\text { De 10 a 49 } \\
\text { empleados }\end{array}$ & $\begin{array}{c}\text { De 50 a 249 } \\
\text { empleados }\end{array}$ & $\begin{array}{c}250 \text { y más } \\
\text { empleados }\end{array}$ & TOTAL \\
\hline Número de empresas del subgrupo producción & 3 & 10 & 8 & 12 & 33 \\
\hline Número de empresas del subgrupo servicios & 27 & 14 & 1 & 1 & 43 \\
\hline TOTAL & 30 & 24 & 8 & 14 & 76 \\
\hline
\end{tabular}

Tabla I. Caracterización de la muestra

\begin{tabular}{|c|c|}
\hline Población & Empresas españolas del sector de las energías renovables (726 empresas) \\
\hline Ámbito geográfico & España \\
\hline Tamaño muestral & 76 empresas \\
\hline Unidad de análisis & Directivos de empresa o unidad de negocio \\
\hline Método de recogida de la información & Cuestionario online y contacto telefónico \\
\hline Tasa de respuesta & $10.47 \%$ (76 de 726) \\
\hline Error muestral & $10.64 \%$ \\
\hline Nivel de confianza & $95 \% ; \mathrm{z}=1.96 ; \mathrm{p}=\mathrm{q}=0.5$ \\
\hline Fecha de realización del trabajo de campo & Septiembre- diciembre 2012 \\
\hline
\end{tabular}

Tabla 2. Ficha técnica de la investigación

ISSN: 07 I8-2724. (http://www.jotmi.org)

Journal of Technology Management \& Innovation (c) Universidad Alberto Hurtado, Facultad de Economía y Negocios. 


\section{Medida de los resultados de innovación en producto $y$ en proceso}

Para medir los resultados de innovación en producto y en proceso se tomaron como referencia las escalas desarrolladas por Zahra y Das (1993), Donate y Guadamillas (2008) y Delgado et al. (20II) (ver la lista de ítems en el anexo). Según Zahra y Das (1993) es recomendable considerar los resultados de innovación tanto en términos absolutos como en términos relativos (p.ej., en comparación con los competidores, periodos anteriores o la media de innovación del sector de actividad). Así, una empresa es innovadora no solamente por el hecho de dedicar esfuerzos a la $1+D+i$, sino por hacerlo en mayor medida que los principales competidores del sector, o con más intensidad que en un período de tiempo anterior.Además, dado que el sector de las energías renovables es bastante heterogéneo, ya que posee ramas de actividad diferentes entre sí, resulta necesario incorporar ítems relativos o comparativos que complementen a los absolutos con el objetivo de ofrecer una imagen más homogénea en términos de resultado innovador.

\section{Variables de control}

La variable tamaño (medida como el logaritmo neperiano del número de trabajadores que posee la empresa) se ha incluido en el modelo como variable de control, ya que las grandes empresas disponen de más recursos y tienen la posibilidad de invertir más en tecnología que las pequeñas (Zahra y Das, 1993; Zahra y Bogner, 1999), lo que nos lleva a pensar que los resultados de innovación podrían verse influidos por la dimensión empresarial.

También se ha considerado como variable de control el tipo de actividad que lleva a cabo la empresa. Para ello se ha dividido la muestra de empresas en dos grandes grupos de actividad: producción y servicios. Consideramos que es importante incluir esta variable en el análisis porque podría condicionar los resultados de innovación obtenidos. Así, con el fin de operativizar la pertenencia a los dos subgrupos considerados, a las empresas incluidas en el grupo de producción se les otorgará un valor uno (variable dummy), mientras que a las empresas comprendidas en el grupo de servicios se les otorgará un valor cero.

\section{Análisis estadístico y discusión de resultados}

El contraste de hipótesis se ha llevado a cabo mediante un modelo de ecuaciones estructurales (SEM). Concretamente, la técnica utilizada ha sido PLS (mínimos cuadrados parciales) mediante el software SmartPLS 2.0, desarrollado por Ringle, Wende y Will (2005).

SmartPLS es un modelo de estimación y análisis SEM que utiliza el proceso de estimación en dos fases. Por un lado se estima el modelo de medida y por otro lado se realiza la estimación del modelo estructural.

\section{Análisis del modelo de medida Fiabilidad de las variables reflectivas}

Los valores de los indicadores de fiabilidad se muestran en la tabla 3, en la cual se observa que tanto los índices de fiabilidad compuesta como los a de Cronbach tienen una alta consistencia interna en los cuatro constructos analizados, ya que ambas medidas superan los niveles recomendados de 0.8 y 0.7 respectivamente (Gefen y Straub, 2005), lo que verifica por tanto la consistencia interna de las variables del modelo.

\section{Validez convergente y discriminante}

Se considera que hay validez convergente cuando los ítems se correlacionan estrechamente con sus constructos teóricos (Gefen y Straub, 2005). Uno de los criterios de validez se determina a través del análisis de las cargas factoriales (Chin, 1998; Tenenhaus et al., 2005) y otro mediante la varianza extraída promedio (AVE). Cuanto mayores son las cargas factoriales, mayor es la evidencia de la unidimensionalidad del constructo. En este trabajo se ha eliminado un indicador de la variable dinamismo percibido del entorno (Dinentl) por no alcanzar el valor mínimo de 0.6 fijado por Falk y Miller (1992). Con respecto al AVE, todos los valores alcanzados superan el valor mínimo recomendado de 0.5 (Fornell y Larcker, 1981; Chin, 1998), por lo que se puede afirmar que los indicadores explican más del cincuenta por ciento de la varianza de sus constructos y por lo tanto existe validez convergente (véase tabla 3 ).

\begin{tabular}{|c|ccc|}
\hline Variables & $\alpha$ de Cronbach & Fiabilidad compuesta (IFC) & AVE \\
\hline Dinamismo percibido del entorno & 0.7999 & 0.8649 & 0.6166 \\
Postura tecnológica & 0.9088 & 0.9359 & 0.7851 \\
Resultados de innovación en producto & 0.8649 & 0.908 & 0.7117 \\
Resultados de innovación en proceso & 0.9138 & 0.9327 & 0.6981 \\
\hline
\end{tabular}

Tabla 3.Análisis del instrumento de medida: fiabilidad y validez convergente

ISSN: 07 I8-2724. (http://www.jotmi.org)

Journal of Technology Management \& Innovation (c) Universidad Alberto Hurtado, Facultad de Economía y Negocios. 
Por otro lado, para que exista validez discriminante en un constructo han de darse correlaciones débiles entre sus indicadores y otros constructos que midan fenómenos diferentes. Ésta se analiza mediante dos métodos indicados por Gefen y Straub (2005). En primer lugar, a través del análisis de las correlaciones cruzadas entre todos los ítems y los distintos constructos (cross-loading). En este caso, podemos afirmar que los cuatro constructos manifiestan mayores correlaciones con sus indicadores de medida que con el resto de indicadores del modelo, lo que indica la existencia de validez discriminante.

El segundo método determina que existe validez discriminante cuando la raíz cuadrada de la varianza extraída promedio (AVE) de la variable latente es mayor que las correlaciones entre dicho constructo y el resto de variables latentes. Así, tal y como se observa en la tabla 4 , los valores en la diagonal principal son superiores a los valores que se encuentran fuera de ella, lo que indica que también en este caso existe validez discriminante.

\section{Análisis del modelo estructural y resultados}

El modelo estructural evalúa el peso y la magnitud de las relaciones entre las distintas variables. La bondad del ajuste del modelo es comprobada a través del análisis del estadístico $t$ de Student, el nivel de significación de los parámetros path y el valor R2 para cada variable dependiente. Así, se aceptarán aquellos coeficientes path, y por extensión las hipótesis planteadas, que sean significativos según una distribución $\mathrm{t}$ de Student de I cola con $\mathrm{n}$ - I grados de libertad (véase tabla 5 y figura I).

\section{Hipótesis}

Cuando se interpretan los coeficientes path, se observa que para las tres hipótesis formuladas éstos superan el valor de 0.3 , demostrándose la existencia de una estrecha relación entre el dinamismo percibido del entorno y la postura tecnológica; y entre ésta y los resultados de innovación, tanto en producto como en proceso.Además, las tres hipótesis se aceptan con un nivel de significación del $\mathbf{9 9 . 9 \%}$, por lo que se puede afirmar, por un lado, que la percepción de un entorno dinámico por parte de los directivos ejerce un efecto significativamente positivo sobre la intención de la empresa de adoptar una postura de liderazgo tecnológico $(\beta=0.376$; $p<0.001$ ) y por otro lado, que cuanto mayor es la tendencia de ésta hacia una postura de liderazgo tecnológico, mayores son también sus resultados de innovación en producto $(\beta=0.579 ; p<0.00 \mathrm{I})$ y en proceso $(\beta=0.505 ; p<0.00 \mathrm{I})$.

En segundo lugar, se analiza la varianza explicada. La bondad de un modelo se determina a través de la fortaleza de cada relación estructural y se analiza utilizando el valor de R2 para cada constructo dependiente. Según Falk y Miller (1992), estos valores deben ser superiores a 0.1 para poder considerar que el modelo tiene suficiente capacidad predictiva. En este estudio, tal y como se puede apreciar en la tabla 6 , el modelo presenta un adecuado poder predictivo, ya que el dinamismo percibido del entorno es capaz de explicar el 14 . $1 \%$ de la varianza de la postura tecnológica adoptada por la empresa, y ésta a su vez explica el $35.7 \%$ de la varianza de los resultados de innovación en producto y el $27.02 \%$ de la varianza de los resultados de innovación en proceso.

\begin{tabular}{|c|c|c|c|c|c|c|}
\hline & Media & $\begin{array}{c}\text { Desviación } \\
\text { típica }\end{array}$ & $\begin{array}{c}\text { Dinamismo } \\
\text { percibido }\end{array}$ & $\begin{array}{c}\text { Innovación en } \\
\text { proceso }\end{array}$ & $\begin{array}{c}\text { Innovación en } \\
\text { producto }\end{array}$ & $\begin{array}{c}\text { Postura tec- } \\
\text { nológica }\end{array}$ \\
\hline \multirow{4}{*}{$\begin{array}{c}\text { Innovamismo } \\
\text { Innovión en proceso } \\
\text { Postura tecnológica }\end{array}$} & 3.62 & 1.18 & 0.7852 & & & \\
\cline { 2 - 7 } & 3.42 & 1.03 & 0.512 & 0.8355 & & \\
\cline { 2 - 7 } & 3.35 & 1.18 & 0.3199 & 0.6214 & 0.8436 & \\
\cline { 2 - 7 } & 4.08 & 1.01 & 0.3761 & 0.5352 & 0.6159 & 0.8861 \\
\hline
\end{tabular}

Tabla 4.Validez discriminante

\begin{tabular}{|c|c|c|c|c|}
\hline Hipótesis & Relación & Coeficientes & Valor t (Boot- & $\begin{array}{c}\text { Contraste de } \\
\text { strap) }\end{array}$ \\
\hline $\mathrm{HI}$ & Dinamismo percibido del entorno $\rightarrow$ Postura tecnológica & $0.376 * * *$ & 3.993 & Se acepta \\
\hline $\mathrm{H} 2$ & Postura tecnológica $\rightarrow$ Resultados de innovación en producto & $0.579 * * *$ & 7.026 & Se acepta \\
\hline $\mathrm{H} 3$ & Postura tecnológica $\rightarrow$ Resultados de innovación en proceso & $0.505^{* * *}$ & 4.946 & Se acepta \\
\hline
\end{tabular}

Tabla 5. Contraste de hipótesis

$$
*_{\mathrm{p}}<0.05(\mathrm{t}(0.05 ; 499)=1.645) ; *^{*} \mathrm{p}<0.01(\mathrm{t}(0.01 ; 499)=2.327) ;{ }^{* * *} \mathrm{p}<0.001(\mathrm{t}(0.00 \mathrm{I} ; 499)=3.092)
$$

ISSN: 07 I8-2724. (http://www.jotmi.org) 
Para valorar el ajuste del modelo en función de la relevancia predictiva, además del estudio del valor R2 para cada variable endógena, se realiza el test de Stone-Geisser (Q2). De acuerdo con Chin (1998), se puede afirmar que la predicción de los constructos es relevante cuando Q2 obtiene valores positivos ( $\mathrm{Q} 2>0)$, tal y como ocurre en este análisis (véase tabla 6).

Finalmente, cabe señalar que la variable tamaño (medida como el logaritmo neperiano del número de trabajadores que posee la empresa) se ha incluido en el modelo como variable de control por ser una variable relevante, pese a la controversia existente cuando se trata de explicar la relación entre innovación y tamaño empresarial (véase por ej. Camisón et al., 2004). Existen investigaciones que señalan una relación positiva entre ambas variables, apuntando el tamaño organizativo como el mejor predictor de la innovación, otras defienden la existencia de una relación nega- tiva y otras incluso señalan la inexistencia de dicha relación (Camisón et al., 2004). Algunos de los que consideran que existe una relación positiva entre ambas variables sostienen que las grandes empresas poseen más recursos para desarrollar su estrategia de innovación y una gran habilidad para obtener mejores resultados derivados de este esfuerzo innovador (Zahra y Das, 1993; Zahra y Bogner, 1999).

En este trabajo hallamos que el tamaño empresarial solo influye significativamente sobre los resultados de innovación en proceso $(\beta=0.186 ; p<0.05)$, siendo no significativa su relación con los resultados de innovación en producto. Así, basándonos en el trabajo de Gibbons y O'Connor (2003) - Bierly y Daly (2007), este hecho podría interpretarse de modo que las innovaciones en proceso adquieren mayor importancia para las grandes empresas que para las pequeñas, pues es probable que contribuyan más que las innovaciones en producto a conseguir economías de escala en

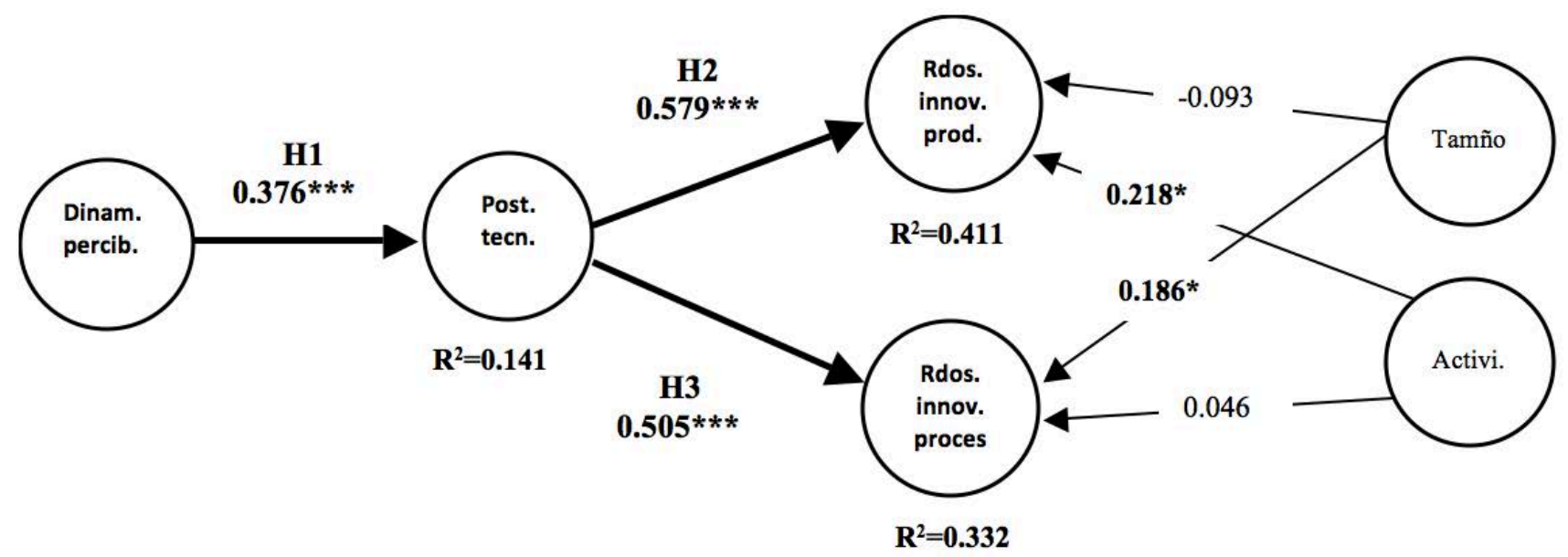

Figura I. Modelo estructural $*_{\mathrm{p}}<0.05(\mathrm{t}(0.05 ; 499)=1.645) ; *^{*} \mathrm{p}<0.01(\mathrm{t}(0.01 ; 499)=2.327) ; *^{* *} \mathrm{p}<0.001(\mathrm{t}(0.001 ; 499)=3.092)$

\begin{tabular}{|c|c|c|c|c|c|}
\hline VARIABLE DEPENDIENTE & $\mathrm{R}^{2}$ & $\mathrm{Q}^{2}$ & $\begin{array}{l}\text { Efecto } \\
\text { directo }\end{array}$ & Correlación & $\begin{array}{l}\text { Varianza } \\
\text { explicada }\end{array}$ \\
\hline Postura tecnológica & 0.141 & 0.1035 & & & \\
\hline HI: Dinamismo percibido $\rightarrow$ Postura tecnológica & & & 0.376 & 0.376 & $14.1 \%$ \\
\hline Resultados de innovación en producto & 0.411 & 0.2839 & & & \\
\hline \multicolumn{6}{|l|}{ H2: Postura tecnológica $\rightarrow$ Resultados innovación producto } \\
\hline & & & 0.579 & 0.616 & $35.7 \%$ \\
\hline Resultados de innovación en proceso & 0.332 & 0.2337 & & & \\
\hline \multicolumn{6}{|l|}{ H3: Postura tecnológica $\rightarrow$ Resultados innovación proceso } \\
\hline & & & 0.505 & 0.535 & $27.02 \%$ \\
\hline
\end{tabular}

Tabla 6. Relevancia predictiva del modelo y efecto sobre las variables dependientes

ISSN: 07 I8-2724. (http://www.jotmi.org) 
producción. Por su parte, las pequeñas empresas, en lugar de desarrollar innovaciones tecnológicas para incrementar la producción y conseguir ventajas competitivas en costes (como suelen hacer las grandes empresas) desarrollarán innovaciones para dar una respuesta más rápida a las demandas del mercado. En este sentido, $y$ teniendo en cuenta que gran parte del tejido empresarial del sector de las energías renovables en España son pequeñas y medianas empresas, esto podría llevarnos a afirmar que las pymes de este sector tienen una mayor tendencia a desarrollar innovaciones en producto y las grandes empresas, por su parte, tienden en mayor medida a desarrollar innovaciones en proceso.

La otra variable de control considerada es el tipo de actividad que lleva a cabo la empresa, por lo que se ha dividido la muestra de empresas en dos grandes grupos de actividad: producción y servicios. Los resultados muestran que las empresas dedicadas a la producción de energía, a la fabricación de componentes tecnológicos y al desarrollo de actividades de ingeniería, soluciones integrales e innovación están positivamente relacionadas con los resultados de innovación en producto $(\square=0.218 ; p<0.05)$, siendo no significativa su relación con los resultados de innovación en proceso. Este hecho puede explicarse porque este grupo de empresas es el encargado de desarrollar componentes tecnológicos y productos tales como paneles solares, turbinas o aerogeneradores. Es lógico pensar que el desarrollo de este tipo de productos esté relacionado con este tipo de empresas y no con las comprendidas dentro del grupo servicios (consultoras energéticas, empresas dedicadas a la comercialización/ exportación de componentes tecnológicos y aquellas que desarrollan estudios/ proyectos y realizan actividades de instalación y mantenimiento). Sin embargo, la inclusión de estas variables de control en el modelo no modifica la significación de las relaciones entre el dinamismo percibido del entorno, la postura tecnológica y los resultados de innovación (en producto y en proceso).

\section{Conclusiones}

La innovación es un concepto clave para las organizaciones de hoy, ya que representa la esencia de sus ventajas competitivas (Fernández-Mesa, Alegre-Vidal y Chiva-Gómez, 2012: 164). Generalmente, las empresas utilizan la tecnología para crear y mantener ventajas competitivas a través de la oferta de productos novedosos, utilizando nuevos procesos, o redefiniendo los límites de su sector de actividad.Así, bajo la perspectiva el EBR, cuando se persigue una postura tecnológica se deben considerar los objetivos de la compañía, su capacidad para gestionar diversas iniciativas referentes a nuevos productos o procesos, su orientación al riesgo, sus recursos disponibles y sobre todo el entorno que rodea a la organización (Zahra, 1996: 190; Bierly y Daly, 2007; Jansen, Vera y Crossan, 2009; Schilke, 20I3).
Desde nuestro punto de vista, la principal contribución de este artículo es la propuesta de una nueva perspectiva sobre la relación entre el dinamismo percibido del entorno y la postura tecnológica de la empresa. En general, este trabajo pretende señalar la importancia que tiene la percepción del dinamismo del entorno por parte de los directivos a la hora de perseguir una postura de liderazgo tecnológico en la empresa, sobre todo en aquellos sectores de actividad intensivos en tecnología, como es el caso del sector de las energías renovables. Por ello, en este trabajo hemos analizado si el mayor o menor grado de dinamismo del entorno percibido por los directivos condiciona la postura tecnológica escogida por la empresa (considerando el dinamismo del entorno como antecedente de la postura tecnológica), en lugar de analizar el efecto que pueda ejercer esta variable sobre la relación entre la postura tecnológica y los resultados de innovación (como han hecho otros trabajos previos que han considerado el dinamismo del entorno como variable moderadora).

Otra novedad en la manera de abordar este trabajo respecto a estudios previos sobre características del entorno es la consideración de las percepciones de los directivos cuando hacemos referencia al dinamismo, ya que este concepto no se analiza desde un punto de vista externo u objetivo, sino que se analiza desde un punto de vista interno de la empresa, más subjetivo y particular de cada directivo. Por lo tanto, considerar este concepto en función de las percepciones particulares de los directivos también propicia que el dinamismo del entorno se introduzca como antecedente de la postura tecnológica perseguida por la empresa. Finalmente, la consideración de la estrategia de innovación tecnológica exclusivamente desde el punto de vista de la postura tecnológica y sin considerar el gasto en $\mathrm{I}+\mathrm{D}+\mathrm{i}$, que tradicionalmente ha sido el indicador por excelencia para medir la estrategia de innovación tecnológica (Spital y Bickford, 1992), es otra novedad de este artículo.

Los resultados de este trabajo muestran que el dinamismo percibido del entorno, tradicionalmente considerado como variable moderadora entre la estrategia de innovación tecnológica y los resultados empresariales (Spital y Bickford, 1992; Zahra y Bogner, 1999; Bierly y Daly, 2007; Jansen, Vera y Crossan, 2009), también puede considerarse una variable exógena capaz de condicionar la postura tecnológica perseguida por la empresa (Zahra, 1996; Su y Chiu, 2004), ya que cuanto mayor sea el grado de dinamismo del entorno percibido por los directivos mayor será la tendencia de la empresa hacia la búsqueda de una postura de liderazgo tecnológico con el objetivo de identificar mercados emergentes, lanzar nuevos productos, adelantarse a la entrada de nuevos competidores o guiar los cambios en el estándar tecnológico, entre otros aspectos. 
Por otro lado, ya hemos visto que los líderes tecnológicos poseen habilidades, competencias tecnológicas acumuladas y capacidades dinámicas que los seguidores no poseen, además de un mayor conocimiento del mercado que éstos y una mayor motivación para actuar (Spital y Bickford, 1992; Haleblian et al., 20I2). Por todo ello, y a la vista de los resultados obtenidos en este trabajo, también podemos afirmar que la adopción de una postura de liderazgo tecnológico en la empresa influye positiva y significativamente sobre los resultados de innovación, tanto en producto como en proceso (Zahra, 1996; Zahra y Bogner, 1999). Sin embargo, nuestros resultados muestran que los líderes tecnológicos del sector de las energías renovables logran en mayor medida innovaciones en producto que en proceso. Hecho que podría justificarse porque la mayor parte del tejido empresarial de este sector son pequeñas y medianas empresas que persiguen el desarrollo de nuevos productos para conseguir ventajas competitivas.

La principal limitación de este trabajo es que se trata de una investigación de carácter transversal, lo que puede provocar que no se recojan efectos que, al diferirse en el tiempo, son importantes para la generación de resultados y la ventaja competitiva en un sentido dinámico (Teece, Pisano y Shuen, 1997; Eisenhardt y Martin, 2000). Aunque este problema se ha intentado remediar, en cierta medida, con la inclusión de retardos ( 3 años) para las variables, los estudios de carácter longitudinal que abarcan periodos de tiempo más amplios son mucho más adecuados para recoger las consecuencias de la búsqueda de una postura de liderazgo tecnológico en la empresa (Min, Kalwani y Robinson, 2006; Haleblian et al., 2012; Hawk, Pacheco-De-Almeida y Yeung, 20I3). Sin embargo, el gran problema de los mismos es la dificultad para acceder a ciertos datos procedentes de fuentes primarias en distintos periodos de tiempo. En nuestro caso, la totalidad de estos datos proceden de fuentes primarias y han sido obtenidos a través de la técnica de la encuesta. Por ello, la transversalidad de la investigación ha sido, en cierta medida, forzada por las características específicas de los datos necesarios para su realización.

Otra limitación adicional surge porque la recogida de datos se ha realizado en un momento de cambios económicos y legales en el sector de las energías renovables, por lo que es probable que los resultados alcanzados puedan haberse visto afectados por este hecho. Por último, debido a la procedencia de los datos y las dificultades para el acceso a los mismos, la muestra analizada no es demasiado amplia, lo que podría afectar a la generalización de los resultados de la investigación.

La propuesta de líneas de investigación futuras tiene, en su mayor parte, una relación directa con las limitaciones señaladas anteriormente. Así, en primer lugar, el desarrollo de investigaciones de carácter longitudinal es un reto importante en el ámbito de la postura tecnológica. Además, la replicación de la investigación en otros sectores o zonas geográficas también sería una opción factible que implicaría la validación de los resultados obtenidos. Asimismo, se podrían incluir otras variables en el modelo tales como la responsabilidad social corporativa, para analizar si un comportamiento socialmente responsable por parte de los líderes tecnológicos del sector puede provocar un incremento de los resultados de innovación en proceso, con el objetivo de mejorar la eficiencia energética y reducir las emisiones de residuos.

\section{Anexo I. Descripción de los ítems utilizados}

Dinamismo percibido del entorno

(I) Los cambios en el entorno de su mercado local han sido intensos.

(2) Sus clientes han solicitado regularmente nuevos productos y servicios.

(3) Se han presentado cambios en su mercado local continuamente.

(4) El número de productos y servicios que han salido al mercado ha cambiado rápida y frecuentemente.

(5) Ha habido pocos cambios (o ninguno) en su mercado.

Postura tecnológica

(I) Importancia concedida por la empresa a comercializar nuevas tecnologías.

(2) Importancia concedida a ser la primera en introducir productos nuevos o mejorados en el mercado.

(3) Importancia concedida a ganarse una reputación en el sector en cuanto a liderar cambios o mejoras en los productos.

(4) Importancia concedida a lograr desarrollar habilidades para introducir nuevos productos o desarrollar nuevos procesos antes que la competencia.

Resultados de innovación en producto

(I) Resultados tecnológicos obtenidos por su empresa en relación al desarrollo de nuevos productos.

(2) Resultados tecnológicos obtenidos en relación a la modificación o mejora de las líneas de producto existente.

(3) Grado de introducción de productos nuevos o mejorados en el mercado, en comparación con sus principales competidores.

(4) Grado de introducción de productos nuevos o mejorados en el mercado en comparación con el anterior periodo de tres años.

Resultados de innovación en proceso

(I) Resultados tecnológicos obtenidos por su empresa en relación al desarrollo de nuevos métodos y procesos de producción.

(2) Resultados tecnológicos obtenidos en relación al desarrollo de mejoras en los métodos y procesos existentes. 
(3) Grado de introducción de métodos y procesos nuevos (o mejorados) en el mercado, en comparación con sus principales competidores.

(4) Grado de introducción de métodos y procesos nuevos (o mejorados) en el mercado, en comparación con el anterior periodo de tres años

(5) Grado de implantación de nuevos procesos que han conseguido reducir el ciclo de fabricación o mejorar la flexibilidad productiva.

(6) Grado de implantación de nuevos procesos que han conseguido reducir los costes de producción.

\section{Referencias}

BIERLY, P.E. y Daly, P.S. (2007). Alternative Knowledge Strategies, Competitive Environment, and Organizational Performance in Small Manufacturing Firms. Entrepreneurship Theory and Practice, 3 I (4), 493-5 I6. DOI: I0.1 I I /j.I5406520.2007.00185.x

BOU-LLUSAR, J.C.; Escrig-Tena, A.B.; Roca-Puig,V. y BeltránMartín, I. (2009). An Empirical Assessment of the EFQM Excellence Model: Evaluation as a TQM Framework Relative to the MBNQA Model. Journal of Operations Management, 27 (I), I-22. DOI: 10.1016/j.jom.2008.04.00 I

BURGELMAN, R.A.; Maidique, M.A. y Wheelwright, S.C. (200I): Strategic Management of Technology and Innovation, McGraw-Hill, Nueva York, $3^{\mathrm{a}}$ Ed.

CAMISÓN-ZORNOZA, C.; Lapiedra-Alcamí, R.; SegarraCiprés, M. y Boronat-Navarro, M. (2004). A Meta-analysis of Innovation and Organizational Size. Organization Studies, 25 (3), 33I-36I. DOI: I0.1 I77/0I70840604040039

CEPEDA, G. (2006). Understanding the link between knowledge management and firm performance: articulating and codifying critical knowledge areas. International Journal of Knowledge and Learning, 2 (3), 238-262.

CHIN,W.W. (1998). Issues and Opinion on Structural Equation Modeling. MIS Quarterly, 22 (I), 7-I6.

COVIN, J.G.; Slevin, D.P. y Heeley, M.B. (1999). Pioneers and Followers: Competitive Tactics, Environment, and Firm Growth. Journal of Business Venturing, I5 (2), I75- 210. DOI: 10.1016/S0883-9026(98)00015-9

DELGADO, M.; Martín, G.; Navas, J.E. y Cruz, J. (20I I). Capital Social, Capital Relacional e Innovación Tecnológica. Una Aplicación al Sector Manufacturero Español de Alta y Mediaalta Tecnología. Cuadernos de Economía y Dirección de la Empresa, I4 (4), 207-22I. DOI: I0.1016/j.cede.20II.04.00 I
DONATE, M.J. y Guadamillas, F. (2008). La Relación entre la Postura Tecnológica de la Empresa y su Estrategia de Conocimiento: Un Análisis de su Efecto en los Resultados Empresariales. Revista Europea de Dirección y Economía de la Empresa, 17 (4), 29-54.

DOUGHERTY, D. (1992). A practice-centered model of organizational renewal through product innovation. Strategic Management Journal, 13, 77-92. DOI: 10.1002/ smj.4250I31007

EISENHARDT, K.; Furr, N.R. y Bingham, C.B. (2010). Microfoundations of Performance: Balancing Efficiency and Flexibility in Dynamic Environments. Organization Science, 2I (6), I263-I273. DOI: http://dx.doi.org//0. I 287/orsc. I 100.0564

EISENHARDT, K. y Martin, J. (2000). Dynamic Capabilities: What are They?. Strategic Management Journal, 2 I (I0-II), II05-II2I.

FALK, R.F. y Miller, N.B. (1992). A Primer for Soft Modeling. University of Akron Press, Akron.

FERNÁNDEZ-MESA, A., Alegre-Vidal, J. y Chiva-Gómez, R. (20I2). Orientación emprendedora, capacidad de aprendizaje organizativo y desempeño innovador. Journal of technology management \& innovation, 7 (2), I57-I70. DOI: http:// dx.doi.org/I0.4067/S07/8-272420I20002000I3

FORNELL, C. y Larcker, D.F. (198I). Evaluating Structural Equation Models with Unobservable Variables and Measurement Error. Journal of Marketing Research, 18 (I), 39-50.

GEFEN, D. y Straub, D. (2005). A Practical Guide to Factorial Validity using PLS-graph:Tutorial and Annotated Example. Communications of the Association for Information Systems, I6 (I), 9I-109.

GIBBONS, P.T.y O'Connor,T. (2003). Strategic posture, technology strategy and performance among small firms. Journal of Enterprising Culture, II (2), I3I-|46. DOI: I0.I I42/ S021849580300008।

GUERRAS, L.A.y Navas, J.E. (2007). La Dirección Estratégica de la Empresa. Teoría y aplicaciones. Thomson Cívitas, Madrid, $4^{\mathrm{a}}$ ed.

HALEBLIAN, J.J.; McNamara, G; Kolev, K. y Dykes, B.J. (20I2). Exploring Firm Characteristics that Differentiate Leaders from Followers in Industry Merger Waves: A Competitive Dynamics Perspective. Strategic Management Journal, 33 (9), I037-I052. DOI: 10.1002/smj. I96 I 
HAWK, A. Pacheco-De-Almeida, G. y Yeung, B. (2013). FastMover Advantages: Speed Capabilities and Entry into the Emerging Submarket of Atlantic Basin LNG. Strategic Management Journal, 34 (I3), I 53 I-I550. DOI: I0. 1002/smj.2085

JANSEN, J.J.P.;Vera, D. y Crossan, M. (2009). Strategic leadership for exploration and exploitation: The moderating role of environmental dynamism. The Leadership Quarterly, 20 (I), 5-I8. DOI: I0.1016/j.leaqua.2008.II.008

LIEBERMAN, M.B. y Montgomery, D.B. (1988). First-mover advantages. Strategic Management Journal, 9, 4I-58. DOI: I0.1002/smj.4250090706

MIN, S.; Kalwani, M.U. y Robinson, W.T. (2006). Market Pioneer and Early Follower Survival Risks: A Contingency Analysis of Really New Versus Incrementally new ProductMarkets. Journal of Marketing, 70 (enero 2006), I5- 33. DOI: http://dx.doi.org/I0.1509/jmkg.2006.70.1.I5

PARKER, A.R. (2000). Impact on the Organizational Performance of the Strategy-Technology Policy Interaction. Journal of Business Research, 47(I), 55-64. DOI: 10.1016/SOI482963(98)0005I-4

PAVITT, K. (1990). What we know about the strategic management of Technology. California Management Review, 31 (3), I7-26.

PODSAKOFF, P.M. y Organ, D.W. (1986). Self-Reports in Organizational Research: Problems and Prospects. Journal of Management, 12 (4), 53I-544. DOI: I0.1 I77/0I492063860I200408

PORTER, M.E. (1985). Competitive Advantage: Creating and Sustaining Superior Performance. The Free Press, Nueva York.

RINGLE, C.M.;Wende, S. y Will, S. (2005). SmartPLS 2.0 (M3) Beta, Hamburg 2005. Disponible en http://www.smartpls.de (acceso 2I/08/20I2).

SCHILKE, O. (20I3). On the Contingent Value of Dynamic Capabilities for Competitive Advantage: The Nonlinear Moderating Effect of Environmental Dynamism. Strategic Management Journal, 35 (2), I 79-203. DOI: I0. I002/smj.2099

SCHILLING, M., 2012. Strategic Management of technological innovation, Mc-GrawHill: NY, $4^{\mathrm{a}}$ ed.

SPITAL, F.C. y Bickford, D.J. (1992). Successful competitive and technology strategies in dynamic and stable product technology environments. Journal of Engineering and Technology Management, 9 (I), 29-60. DOI: 10.1016/09234748(92)90010-3
SU, Y.S. y Chiu, H.J. (2004). Determinants of Biotechnology Ventures' Strategic Choice: Firm Capability, Environmental Dynamism and Partnering Relations. Paper submitted to 2004 Asia Academy of Management, Shanghai, China, I-3 I.

SUÁREZ, F.F. (2004). Battles for Technological Dominance: An Integrative Framework. Research Policy, 33 (2), 27I-286. DOI: 10.1016/j.respol.2003.07.00I

SUAREZ, F.F.y Lanzolla, G. (2007).The role of environmental dynamics in building a first mover advantage theory. Academy of Management Review, 32 (2), 377-392. DOI: 10.5465/ AMR.2007.24349587

TEECE, D.J., Pisano, G. y Shuen, A. (1997). Dynamic Capabilities and Strategic Management. Strategic Management Journal, 18 (7), 509-533.

TENENHAUS, M.; Vinzi, V.E.; Chatelin, Y. y Lauro, C. (2005). PLS Path Modeling. Computational Statistics \& Data Analysis, 48 (I), 159-205. DOI: 10.1016/j.csda.2004.03.005

WIJBENGA, F.H. y Van Witteloostuijn, A. (2007). Entrepreneurial Locus of Control and Competitive Strategies- The Moderating Effect of Environmental Dynamism. Journal of Economic Psychology, 28 (5), 566- 589. DOI: 10.1016/j. joep.2007.04.003

WILBON, A.D. (2003). Competitive posture and IPO performance in high technology firms. Journal of Engineering and Technology Management, 20 (3), 23 I-244. DOI: I0.1016/ S0923-4748(03)00020-I

WILDEN, R., Gudergan, S.P., Nielsen, B.B. y Lings, I. (20I3). Dynamic capabilities and performance: strategy, structure and environment. Long Range Planning, 46 (I), 72-96. DOI: 10.1016/j.Irp.2012.12.00I

YANG, J. y Liu, C.Y. (2006). New product development: An innovation diffusion perspective. The Journal of High Technology Management Research, 17 (I), 17-26. DOI: 10.1016/j. hitech.2006.05.002

ZAHRA, S.A. (1996). Technology Strategy and Financial Performance: Examining the Moderating Role of the Firm's Competitive Environment. Journal of Business Venturing, II (4), I89-2 I9. DOI: I0.10I6/0883-9026(96)0000I-8

ZAHRA, S.A.y Bogner,W.C. (1999). Technology Strategy and Software New Ventures' Performance: Exploring the moderating Effect of the Competitive Environment. Journal of Business Venturing, I5 (2), 135-173. DOI: 10.1016/S08839026(98)00009-3

ISSN: 07 I8-2724. (http://www.jotmi.org) 
ZAHRA, S.A. y Das, S.R. (1993). Innovation Strategy and Financial Performance in Manufacturing Companies: An Empirical Study. Production and Operations Management, 2 (I), 15-37. DOI: 10.1 III/j.1937-5956.1993.tb00036.x

INFORME APPA (20II): Estudio del Impacto Macroeconómico de las Energías Renovables en España, pp. I-I40. 